\title{
A REView ON BLIND STILl IMAge STEganalysis TECHNIQUES USINg FEATURES EXTRACTION AND Pattern Classification Method
}

\author{
Dr. Monisha Sharma ${ }^{1}$ and Mrs. Swagota Bera ${ }^{2}$ \\ ${ }^{1}$ Department of Electronics \& Telecommunication ,CSVT University,Bhilai \\ monisha.sharma10@gmail.com \\ ${ }^{2}$ Department of Electronics \& Telecommunication,CSVT University,Bhilai \\ swagotaberasarkar@gmail.com
}

\begin{abstract}
Steganography is the technique for hiding secret information in other data such as still, multimedia images, text, audio. Whereas Steganalysis is the reverse technique in which detection of the secret information is done in the stego image. Steganalysis can be classified on the basis of the techniques used classified statistical techniques, pattern classification techniques and visual detection techniques. All the existing techniques can be broadly classified on the basis of the information required for the designing of the steganalysis. They are targeted and blind steganalysis In targeted technique, we first look at steganalysis techniques is designed for a particular steganographic embedding algorithm in mind whereas in blind steganalysis is general class of steganalysis techniques which can be implemented with any steganographic embedding algorithm, even an unknown algorithm. In this paper, an extensive review report is presented chronologically on the Blind Image Steganalysis for the still stego images using the classification techniques.
\end{abstract}

\section{KEY WORDS}

Steganography, Steganalysis, Cover image, Stego image, cover Image, Attack, Least Significant Bit (LSB), DCT,WT, FFT, SVM, GA, ANN, ANOVA

\section{INTRODUCTION}

Hidden in the X-rated pictures on several pornographic Web sites and the posted comments on sports chat rooms may lay the encrypted blueprints of the next terrorist attack against the United States or its allies. It sounds farfetched, but U.S. officials and experts say it's the latest method of communication being used by Osama bin Laden and his associates to outfox law enforcement. Bin Laden, indicted in the bombing in 1998 of two U.S. embassies in East Africa, and others are hiding maps and photographs of terrorist targets and posting instructions for terrorist activities on sports chat rooms, pornographic bulletin boards and other Websites, U.S. and foreign officials say[1].

Above is reported in USA TODAY AP by Jack Kelley brought the world's attention to the use of steganography. Greater still was the impact to come from the devastation on the 11 September 2001 of the World Trade Centre. The result of this destructive act also consistently motivating us to explore in the secret data detection.

Steganalysis is the art of Science which deals with the detection \& destruction of the secret image. Various approaches are discussed by the different researchers in the area of steganalysis 
.Broadly, there are two approaches to the problem of steganalysis, and one is to come up with a steganalysis method specific to a particular steganographic algorithm known as embedding Algorithm based steganalysis Techniques. For decoding we must known the encoding algorithm. Complete recovery of the secret data is possible. The other technique is more general class of steganalysis techniques pioneered independently can be designed to work with any steganographic embedding algorithm, even an unknown algorithm. Such techniques have subsequently been called Universal Steganalysis techniques or Blind Steganalysis Techniques. A steganalysis technique specific to an embedding method would give very good results when tested only on that embedding method, and might fail on all other steganographic algorithms. But blind steganalysis is the universal one which can be implemented to any stego image.

Such techniques essentially design a classifier based on a training set of cover-objects and stegoobjects arrived at from a variety of different algorithms. Classification is done based on some inherent. Features of typical natural images which can get violated when an image undergoes some embedding process .Hence, designing a feature classification based universal steganalysis technique consists of tackling two independent problems. The first is to find and calculate features which are able to capture statistical changes introduced in the image after the embedding process. The second is coming up with a strong classification algorithm which is able to maximize the distinction captured by the features and achieve high classification accuracy. Typically, a good feature should be accurate, consistent and monotonic in capturing statistical signatures left by the embedding process. Prediction accuracy can be interpreted as the ability of the measure to detect the presence of a hidden message with minimum error on average. Similarly, prediction monotonicity signifies that the features should ideally be monotonic in their relationship to the embedded message size. Finally, prediction consistency relates to the feature's ability to provide consistently accurate predictions for a large set of steganography techniques and image types. This implies that the feature should be independent on the type and variety of images supplied to it .Embedding techniques affect different aspects of images.

Different approaches are given by the various researchers for feature extraction from images. The authors argue that most of the specific steganalysis techniques concentrate on first order statistics, i.e. histogram of DCT coefficients. Quadratic mirror filters (QMF) are used to decompose the image, after which higher order statistics such as mean, variance, kurtosis, and skewness are calculated for each subband. Also the error obtained from an optimal linear predictor of coefficient magnitudes of each sub band is used as a second set of features. In all the above methods, the calculated features are used to train a classifier, which in turn is used to classify clean and stego images. Different classifiers have been employed by different authors; Ascribes uses a MMSE Linear predictor, where as Farad uses a Fisher linear discriminate and also a Support Vector Machine (SVM) classifier. SVM classifiers seem to have much better performance in terms of classification accuracy compared to linear classifiers since they are able to classify non-linearly separable features $[2,3,4]$.

Steganalysis can also be classified based on the detection parameters .If the detection is done in the basis of the differences in the visual texture of the stego image and cover image then known as visual attacking whereas if the detection is done in the basis of the variation in the statistical parameters of the stego image and cover image the known as the statistical attacking. These attacking technique need the information of cover image and stego image. The universal steganalysis is better technique in which the technique can be implemented to any randomly incoming images and detection can be done. Any portion of the incoming image may alter after applying the detection algorithm. If after removing the noise effect the alteration remains then it is detected that the secret data is hidden in it. If the detection result indicate the presence of hidden information, then the analytic will try to recover the secret data by applying the decoding algorithm in the hit and trial method. Prediction accuracy can be interpreted as the 
International Journal of Computer Science, Engineering and Information Technology (IJCSEIT), Vol.2, No.3, June 2012

ability of the measure to detect the presence of a hidden message with minimum error on average. The feature should be independent on the type and variety of images supplied to it $[5,6,7]$.

The steganography and steganalysis has already discussed in the previous survey report [8]. Few Image Statistical and visual steganalysis techniques are implemented and discussed in previous paper $[9,10]$.

\section{BLIND IMAGE STEGANALYSIS TECHNIQUES}

In this section, a few proposed techniques for blind image steganalysis for the JPEG and nonJPEG stego images, based on the classification techniques which will improve the detection accuracy of the secret has been introduced.

\section{Steganlysis Based on Markov Model of Thresholded Prediction -Error Image (2006)}

Dekun Zou, Yun Q. Shi1, Wei Su and Guorong Xuan[11] proposed a steganalysis system based on 2-D Markov chain of thresholded prediction-error image. Image pixels are predicted with their neighboring pixels, and the prediction-error image is generated by subtracting the prediction value from the pixel value and then thresholded with a predefined threshold. The empirical transition matrixes of Markov chain along the horizontal, vertical and diagonal directions serve as features for steganalysis. Support vector machines (SVM) are utilized as classifier

\section{Steganalysis Embedding Percentage Determination with Learning Vector Quantization (2006)}

B.M Rodriguez, G.L. Peterson, K.W.Bauer and S.S Agaian [12] demonstrated the effectiveness of learning vector quantization (LVQ) as a clustering technique which assists in distinguishing stego and non stego images. Seven features are used over a small set of 200 observations with varying levels of embedded information from $1 \%$ to $10 \%$ in increments of $1 \%$. It also provides a simple method for determining the percentage of embedding given low information embedding percentages.

\section{Blind Image Steganalysis Based on Statistical Analysis of Empirical Matrix (2006)}

Chen Xiaochuan, Wang Yunhong, Tan Tieniu and Guo Lei [13] proposed a novel steganalysis method based on statistical analysis of empirical matrix (EM) is proposed to detect the presence of hidden message in an image. The projection histogram of EM is used to extract features composed of two parts: the moments of $\mathrm{PH}$ and the moments of the characteristic function of $\mathrm{PH}$. Also, features extracted from prediction-error image are included to enhance performance. SVM is utilized as classifier.

\section{Spatial-frequency Feature Vector Fusion Based Steganalysis (2006)}

Cai Hong and S.S Agaian [14] present an algorithm for breaking the JPEG based steganographical algorithms such as F5. First, the proposed method extracts features from spatial domain and DCT domain respectively. Second, the data fusion technique is employed to combine their features. Finally, SVM is used to classify the stego images and non-stego images based on the combined features.

\section{Blind Steganalysis Using wavelet statistics and ANOVA (2007)}

Shuang - Huan Zhan and Hong-Bin Zhang [15] implemented blind steganalysis, statistical model based on high-order wavelet decomposition to capture statistical difference between cover images 
and stego images. However, not all wavelet statistics are able to reflect well statistical changes due to hidden message embedded. Analysis of Variance (ANOVA) is applied to test the wavelet statistics which are more sensitive to hidden message are selected as image's features. Support Vector Machine (SVM) used as classifier.

\section{Passive Steganalysis Using Image Quality Metrics and Multi-class Support Vector Machine (2007)}

Bo Xu, Jiazhen Wang, Xiaqin Liu and Zhe Zhang [16] proposed a scheme for detection of hidden data as well as identifying the hiding domain .Firstly; stenographic domains are classified into four, i.e. spatial domain, DCT domain, DWT domain and ICA domain. Then analyze total 26 image quality measures summarized by Ismail Avcibas and choose eight sensitive features based on the analysis of variance technique as feature set to distinguish between cover-images and stego-images which are marked in the four domains respectively. The classifier between cover and stego-images is built using multi-class support vector machine on the selected feature set.

JPEG Steganalysis based on classwise non principal components analysis and multidirectional Markov Model (2007)

Xuan Guorong, Cui Xia ,Y.Q Shi, Chen Wen, Tong Xuefeng and Huang Cong [17] derived 360 dimensional feature vectors sensitive to data embedding process are derived from multidirectional Markov models in the JPEG coefficients domain. The class-wise non-principal components analysis (CNPCA) is proposed to classify steganography in the high-dimensional feature vector space.

\section{A JPEG Steganalysis Algorithm with embedded rate estimation based on Multi-Class SVM (2008)}

Xiaosheng Pan [18] uses the difference of DCT coefficients of natural-images and stego-images as the feature vector to determine if a hiding message is in the image. Through the analysis of former feature vector, the author extracts a new feature to estimate the amount of hidden information in JPEG.

Image Steganalysis Based on Statistical Moments of Wavelet Subband Histograms in Different Frequencies and Support Vector Machine (2008)

Mohammad Ali Mehrabi [19] proposed a new image Steganalysis scheme based on statistical moments of histogram of multi-level wavelet subbands in frequency domain. Different frequencies of histogram have different sensitivity to various data embedding. Then we decompose the test image using three-level Haar discrete wavelet transform (DWT) into 13 subbands (here the image itself is considered as the LL0 subband).The DFT of each subband, is calculated. It is divided into low and high frequency bands. The first three statistical moments of each band are selected to form a 78-dimensional feature vector for Steganalysis. Support Vector Machines (SVM) classifier is then used to discriminate between stegoimages and innocent images. Experimental results show that the proposed algorithm outperforms previously existing techniques.

\section{A Steganalysis Method Based on Quantization Attack (2008)}

Jifeng Huang Qingju Jiao [20] developed a technique based on one-class SVM for discriminating between cover-images and stegoimages based on the statistical irregularities that distinguish the cover images from stego images. The statistical model consists of detail sub-band coefficients mean squared error. 
International Journal of Computer Science, Engineering and Information Technology (IJCSEIT), Vol.2, No.3, June 2012

A Blind Image Steganalysis Based on Features from Three Domains (2008)

Yuan Liu, Li Huang, Ping Wang Guodong Wang [21] proposed a new blind in which, Robert gradient energy in pixel domain, variance of Laplacian parameter in DCT (discrete cosine transform) domain and higher-order statistics extracted from wavelet coefficients are used to derive the feature vector of the proposed steganalysis algorithm, and BP (back propagation) neural network is applied as the classifier in this paper.

\section{JPEG Steganalysis Using Color Correlation and Training on Clean Images Only (2008)}

Hong Cai; Agaian, S.S. [22] proposed a novel predictor based on the intra- and inter- color correlations of wavelet coefficients. This method achieves higher detection rates, under a blind condition that only involves clean images at the training stage.

\section{A New Steganalysis Method Using High-pass Filter for JPEG Image (2008)}

Han-ling Zhang; Shu-yi Wang[23] extracted the moments in frequency domain of wavelet subband's histogram to analyze the difference of histogram between cover image and stego image, at the same time, used high-pass filter to strengthen the image and then Support Vector Machine (SVM) was used to classify the extracted 40 dimensional feature vectors.

\section{Steganalysis Based on Co-occurrence Matrix of Differential Image (2008)}

Ziwen Sun; Maomao Hui; Chao Guan[24] calculates the forward difference in three directions, horizontal, vertical and diagonal, towards adjacent pixels to obtain three-directional differential images for a natural image. Then the differential images are thresholded with a pre-set threshold to remove the redundant information. The co-occurrence matrixes of thresholded differential images are used as features for steganalysis. Support vector machine (SVM) with RBF kernel are applied as classifier.

Steganalysis of LSB Matching Based on Co-Occurrence Matrix and Removing Most Significant Bit Planes (2008)

Mehrabi, M.A.; Aghaeinia, H.; Abolghasemi, M. [25] derived feature vectors from co-occurrence matrix in spatial domain, which is sensitive to data embedding process. This matrix is derived from an image that some of its most significant bit planes are removed. By this preprocessing in addition to decrease the size of feature vector also preserve effects of embedding. SVM is used for classification.

\section{Image Universal Steganalysis Based on Wavelet Packet Transform (2008)}

Xiangyang Luo; Fenlin Liu; Jianming Chen; Yining Zhang [26] ,firstly, decompose image into three scales through WPT to obtain 85 coefficient subbands together, and extract the multi-order absolute characteristic function moments of histogram from them as features. And then, normalize these features and combine them to a 255-D feature vector for each image. Lastly, according to this vector, a back-propagation (BP) neural network is designed to classify cover and stego images.

\section{Features-Pooling Blind JPEG Image Steganalysis (2008)}

Chiew Kang Leng [27] employed features-pooling method to extract the steganalytic features and the classification is done by using neural network. 
International Journal of Computer Science, Engineering and Information Technology (IJCSEIT), Vol.2, No.3, June 2012

Blind Image Steganalysis Based on Run-Length Histogram Analysis (2008)

Jing Dong and Tieniu Tan [28] higher-order statistics of characteristic functions of three types of image run-length histograms are selected as features. Support vector machine is used as classifier.

\section{Detection of BPCS-Steganography Using SMWCF Steganalysis and SVM (2008)}

Julio, Raul, Mariko and Kazuhiko [29] proposed the steganalysis based on statistical moments of wavelet characteristic function (SMWCF) and artificial neural network (ANN) as classifier.

\section{Steganalysis of LSB Matching Based On Histogram Features in Grayscale Image(2008)}

Xu Mankun, Li Tianyun, Ping Xijian [30] modeled the matching embedding as a kind of image degradation with certain additive pulse noise proportional to the embedding rate. We get the restored image by wavelet denoising as the cover image's estimation. Then we extract the features of 1D statistical grayscale histogram of test images and estimated images to train and classify using SVM.

\section{A New Blind Steganalysis Method for JPEG Images (2008)}

Xiang LI, Shanghai Jiao [31] proposed the steganalysis method based on frequency features from DCT coefficients. This method does not require any training and can be widely used on JPEG images from various sources.

\section{Multiclass Steganalysis for JPEG Stego Algorithms (2008)}

Ping Wang, Fenlin Liu, Guodong Wang, Yifeng Sun, Daofu Gong[32] improves Xuan's universal steganalysis is improved to distinguish cover and stego images by the means of applying Bhattacharyya distance to select the most important features.

\section{Universal JPEG Steganalysis Based on Microscopic and Macroscopic Calibration (2008)}

Fangjun Huang, Bin Li, Jiwu Huang [33] exploits both the magnitude and sign dependencies existed in intra-block and inter-block quantized DCT (discrete cosine transform) coefficients by the Markov empirical transition matrices. Moreover, a new microscopic and macroscopic calibration method is proposed to calibrate the local and global distribution of the quantized DCT coefficients of the test image, thus improve the detecting performance.

\section{Detect Information-Hiding Type and Length in JPEG Images by Using Neuro-fuzzy Inference Systems (2008)}

Liu, Qingzhong; Sung, Andrew H.[34] used the polynomial fitting and computational intelligence techniques based on the Generalized Gaussian Distribution (GGD) model in the quantized DCT coefficients, the errors between the logarithmic domain of the histogram of the DCT coefficients and the polynomial fitting are extracted as features to detect the adulterated JPEG images and the untouched ones. Computational intelligence techniques such as Support Vector Machines (SVM), neuro-fuzzy inference system, etc. are utilized.

Steganalysis of Multi-class JPEG Images Based on Expanded Markov Features and Polynomial Fitting (2008)

Qingzhong Liu; Sung, A.H.; Ribeiro, B.M.; Ferreira, R.[35] calculates the difference of the expanded Markov features between the testing image and the calibrated version, and combine 
International Journal of Computer Science, Engineering and Information Technology (IJCSEIT), Vol.2, No.3, June 2012

these difference features and the polynomial fitting features on the histogram of the DCT coefficients as detectors.

\section{Universal Steganalysis Using Color Correlation and Feature Fusion (2008)}

Yuan-lu Tu; Sheng-rong Gong [36] calculated the features from the luminance and chrominance components of the images. Instead of using single wavelet high-order statistics for luminance component of the image, it extracts features both in DCT and DWT domains, and substitute the wavelet high-order statistics with the moments of wavelet characteristic function which is more sensitive to steganography and implements nonlinear SVM classification.

\section{An Investigation of Genetic Algorithm on Steganalysis Techniques (2009)}

Xiao Yi Yu; Aiming Wang[37] proposed a feature selection and transformation approach for universal steganalysis based on genetic algorithm (GA) and higher order statistics. Three types of typical statistics as candidate features and twelve kinds of basic functions as candidate transformations. The GA is utilized to select a subset of candidate features, a subset of candidate transformations and coefficients of the logistic regression model for blind image steganalysis. The logistic regression model is then used as the classifier.

\section{Feature-Based Steganalysis for JPEG Images (2009)}

Zhuo Li; Kuijun Lu; Xianting Zeng; Xuezeng Pan[38] collected thirteen statistics in the DCT domain and spatial domain and an 82-dimensional feature vector for each image is calculated by using the characteristic function and the center of mass (COM) for each statistic, we calculate. Support vector function (SVM) is utilized to construct the blind classifier.

\section{Universal Image Steganalysis Based on Wavelet Packet Decomposition and Empirical Transition Matrix in Wavelet Domain (2009)}

Xiaoyuan Yang; Yu Lei; Xiaozhong Pan; Jia Liu [39] firstly, decomposes the test image using two-level Haar WPD. The statistical analysis is made both for the test image and their wavelet packet subbands. Secondly, it exploits the interscale and intrascale dependencies between wavelet coefficients. Markov empirical transition matrices are used to capture these dependencies. Fisher linear discriminator (FLD) is applied as classifier.

\section{Intelligent Detection of LSB Stego Anomalies in Images Using Soft Computing Paradigms (2009)}

Geetha, S.; Sindhu, S.S.; Ishwarya, N.; Mohan, A.; Amuthayazhini, P.; Kamaraj, N.[40] uses the 27 features that are calculated from the three different statistical moments i.e., PDF, CF and Absolute moment calculated from wavelet multi-resolution representation of the images. Four soft computing techniques viz., Support Vector Machine, NaiÂive Bayes classifier, Decision Tree Classifier and K-nearest neighbor classifier.

\section{Method Based on Characteristic Function Moments of Wavelet Subbands (2009)}

Ziwen Sun; Hui Li; Zhijian Wu; Zhiping Zhou[41]proposed a scheme based on the characteristic function moments of three-level wavelet subbands including the further decomposition coefficients of the first scale diagonal subband. The first three order statistical moments of each band are selected to form a feature vector for steganalysis. The Euclidean distance is used as the separability criterion to analysis the effectiveness of feature vectors for classification and the BP neural network is adopted as the classifier. 
International Journal of Computer Science, Engineering and Information Technology (IJCSEIT), Vol.2, No.3, June 2012

JPEG Image Steganalysis Method Based on Binary Similarity Measures (2009)

Jing-Qu Lin; Shang-Ping Zhong[42] captured the seventh and eighth bit planes of the non-zero DCT coefficients from JPEG images and computes 14 features of each image based on binary similarity measures. These features are used to construct a support vector machine classifier.

\section{Steganalysis Based on Regression Model and Bayesion Network (2009)}

Xiao Yi Yu; Aiming Wang[43]proposed a feature generation and classification approach for universal steganalysis based on genetic algorithm (GA) and higher order statistics. The GA is utilized to select a subset of candidate features, a subset of candidate transformations to generate new features. The logistic regression model and Bayesian network model are then used as the classifier.

\section{Steganalysis for JPEG Images Based on Manifold Learning (2009)}

Xiaomei Quan; Hongbin Zhang [44] extracted the feature by the nonlinear dimensionality reduction method (ISOMAP), which will greatly reduce the dimensionality of the feature space without influencing the performance of our steganalyzer.

Using Higher Order DCT Difference to Effective Improve Markov Process Based JPEG Steganalysis Detection Rate (2009)

Guibin Yang; Hongbin Zhang[45] improves the Markov Process based approach by using several variants and experimented including using JPEG 1D array, using higher order difference of JPEG DCT coefficients, using inter-block 1D array and separating the DCT coefficients to several groups. Support vector machines (SVM) are utilized as classifier.

\section{Reduction of Markov Extended Features in JPEG Image Steganalysis (2009)}

Jing-qu Lin; Xiao-dong Wang; Shang-ping Zhong [46] combined the forward selection algorithm with F-score method to select the Markov extended features. We then compress those selected features to get a smaller feature set according to their directions. Therefore, the dimensionality of feature space is reduced from 324 to 26.

\section{Steganalysis on Character Substitution Using Support Vector Machine (2009)}

Xinxin Zhao; Liusheng Huang; Lingjun Li; Wei Yang; Zhili Chen; Zhenshan Yu[47] used Support Vector Machine (SVM) as a classifier to classify the characteristic vector input into SVM derived using character substitution in texts.

Universal Steganalysis Based on Statistical Models Using Reorganization of Block-based DCT Coefficients (2009)

Shaohui Liu; Lin Ma; Hongxun Yao; Debin Zhao [48] proposed that the block-based DCT by proper reorganization of its coefficients can have similar characteristics to wavelet transforms. The presented universal steganalysis method utilizes these characteristics to build statistical models of the image and its prediction-error image. Features extracted from the re-organization DCT blocks of host images and theirs prediction-error images and features extracted from stego images and theirs prediction-error images are used to train the SVM classifier. 
Steganalysis Based on Bayesion Network and Genetic Algorithm (2009)

Xiao Yi Yu; Aiming Wang[49]proposed a feature selection and transformation approach for universal steganalysis based on Genetic Algorithm (GA) and higher order statistics. We choose three types of typical statistics as candidate features and twelve kinds of basic functions as candidate transformations. The GA is utilized to select a subset of candidate features, a subset of candidate transformations and coefficients of the Bayesion Network Model for blind image steganalysis. The Bayesion Network Model is then used as the classifier.

\section{Blind Steganalysis Method for BMP Images Based on Statistical MWCF and F-score Method (2009)}

Xue Zhang; Shang-Ping Zhong[50] calculated the redundant features by combining wavelet characteristic functions MWCF and F-score method called as FS-MWCF.

\section{A Methodology of Steganalysis for Images (2009)}

Hernandez-Chamorro, A.; Espejel-Trujillo, A.; Lopez-Hernandez, J.; Nakano-Miyatake, M.; Perez-Meana, H.[51]proposed a global steganalysis methodology by comparing some of the steganalysis methods. The secret message detection capacities of these steganalysis methods are evaluated using stegoimages generated by typical data hiding algorithms. The evaluation of steganalysis methods is realized in terms of false negative and false positive error rates using 100 images.

\section{Alpha-Trimmed Image Estimation for JPEG Steganography Detection (2009)}

Mei-Ching Chen; Agaian, S.S.; Chen, C.L.P.; Rodriguez, B.M.[52] introduced an image estimation technique utilizing the alpha-trimmed mean for distinguishing clean and steganography images. The alpha-trimmed method estimates steganographic messages within images in the spatial domain and provide flexibility for classifying various steganography methods in the JPEG compression domain.

\section{Blind Steganalysis Based on Features in Fractional Fourier Transform Domain (2009)}

Chao-en Zhou; Jiu-chao Feng; Yi-xian Yang[53] extracted two kinds of features of an image based on the good property of fractional Fourier transform (FRFT) coefficients of image histogram and the histogram of image FRFT coefficients.SVM is used as a classifier.

\section{Blind JPEG Steganalysis Based on Correlations of DCT Cofficients in Multi-directions and Calibrations (2009)}

Wang Yong; Liu JiuFen; Zhang WeiMing [54] firstly, a new kind of transition probability matrix is constructed to describe correlations of the quantized DCT coefficients in the multi-directions and secondly, by merging two different calibrations, a 96-dimensional feature vector is extracted, and then the SVM is trained to build the steganalyzer.

\section{A Novel Universal Steganalyser Design: "LogSv" (2009)}

Gul, G.; Kurugollu, F.[55] attacked spatial domain steganographic algorithms by using the basic idea behind the proposed method which is twofold: The first is the capability of modeling linear dependencies of image rows/columns in local neighborhoods and the second is the normalization of different energy levels for different images. This idea is simply realized with integrating the 
logarithms of singular values calculated over image sub blocks resulting to a novel staganalyser, named as "LogSv".

\section{Steganalysis for Markov Feature of Difference Array in DCT Domain (2009)}

Zhiping Zhou; Maomao Hui [56] used zigzag scanning pattern is developed to arrange both DCT (Discrete Cosine Transform) blocks and coefficients in each block. Threshold and truncation techniques are utilized to make the computational complexity of the proposed method manageable. Bidirectional Markov matrix is exploited to capture the correlations between the adjacent coefficients in both intra-block and inter-block senses, which have been changed during data embedding. Features for steganalysis are derived from intra-block and inter-block Markov transition matrixes.

\section{A Universal Steganalysis Using Features Derived from the Differential Image Histogram in Frequency Domain (2009)}

Deng Qian-lan; Lin Jia-jun [57] proposed an image steganalysis scheme based on the differential image histogram in frequency domain. The difference is calculated in three directions, horizontal, vertical and diagonal towards adjacent pixels to obtain three-directional differential images for a natural image. Then the features for steganalysis are extracted from the DFT of the histogram of differential images and divided into low and high frequency bands. Support vector machine (SVM) with RBF kernel is applied as classifier.

\section{Universal Steganalysis to Images with WBMC Model (2009)}

Xiaoyuan Yang; Shifeng Wang; Jia Liu [58] used Wavelet based Markov Chain (WBMC) model for nature images, which can present statistic divergence between cover image and steg image prominently. Based on Markov chain empirical matrix, the difference between low frequency domain and high frequency domain generalized by steg process is discussed, and then defined two models: WBMC_L model and WBMC_H model respective to construct our WBMC model.

\section{Blind Detection for JPEG Steganography (2010)}

Wenqiong Yu; Zhuo Li; Lingdi Ping [59] constructed nine statistical models from the DCT and decompressed spatial domain for a JPEG image. By calculating the histogram characteristic function ( $\mathrm{HCF}$ ) and the center of mass (COM), the energy distribution of each model as one part of our feature set is measured. Support vector machines are utilized to construct classifiers.

\section{Statistical Steganalyis of Images Using Open Source Software(2010)}

Kaipa, B.; Robila[60] employed Weka, a data-mining tool developed in java for comparing classification success and error rates and also developed an application using Weka Java library for loading the data of the Images and classify the images .

\section{Block-based Image Steganalysis: Algorithm and Performance Evaluation (2010)}

Seongho Cho; Byung-Ho Cha; Jingwei Wang; Kuo[61] classify the image blocks into multiple classes on steganalysis results of decomposed image blocks and find a classifier for each class to decide whether a block is from a cover or stego image. Consequently, the steganalysis of the whole image can be conducted by fusing steganalysis results of all image blocks through a voting process. 
Block-based Image Steganalysis for a Multi-Classifier (2010)

Seongho Cho; Jingwei Wang; Kuo[62] design a multi-classifier which classifies stego images depending on their steganographic algorithms in addition to distinguishing stego images from cover images based on steganalysis results of decomposed image blocks.

\section{An Ensemble of Classifiers Approach to Steganalysis (2010)}

Bayram, S.; Dirik, A.E.; Sencar, H.T.; Memon [63] firstly, proposed approach provides a workable and systematic procedure to incorporate several steganalyzers together in a composite steganalyzer to improve detection performance in a scalable and cost-effective manner. Secondly, since the approach can be readily extended to multi-class classification it can also be used to infer the steganographic technique deployed in generation of a stego-object.

\section{Steganalysis Algorithm Based on the D Reduction of Improved Differential Matrix in Images (2010)}

Wenqiong Yu; Zhuo Li; Lingdi Ping [64] proposed an SADRID-I image steganalysis algorithm that is based on improved differential matrix, according to the high dimensions and correlation of image features. Using attribute significance and differential matrix that stems from rough sets theory, the algorithm can implement $\mathrm{D}$ attributes reduction to high dimensional statistic features extracted from test image to get more accurate rough features. By screening and extraction, it reduces feature dimension and computational complexity, and correlations between eigenvectors are scientifically counteracted, with an improvement in detection efficiency of information-hiding blind detection system and SVM classifier is employed.

\section{The Blind Detection of Information Hiding in Color Image (2010)}

Deng Qian-lan[65] on the statistic moments of differential image in frequency domain towards the 24 bits color images is calculated in three directions, horizontal, vertical and diagonal towards adjacent pixels to obtain three-directional differential images for a color image. Then the features for steganalysis are extracted from the DFT of the 2D histograms of differential images. Support vector machine with RBF kernel is applied as classifier.

\section{Steganalytic Attack for an Adaptive Steganography Using Support Vector Machine (2010)}

Yamini, B.; Sabitha [66] calculated the length of embedded message using Support Vector Machine to classify the cover and stego images with extremely low computational cost of comparing the bit positions.

\section{Steganography Detection using Localized Generalization Error Model (2010)}

Zhi-Min He; Ng, W.W.Y.; Chan, P.P.K.; Yeung [67] proposed a Radial Basis Function Neural Network (RBFNN) optimized by the Localized Generalization Error Model (L-GEM) for steganograhpy detection. , the discrete cosine transform (DCT) features and the Markov features are used as inputs of neural networks for detection and to enhance the generalization capability of the RBFNN and the performance of detecting steganography in future images, the architecture of the RBFNN is selected by minimizing the L-GEM.

\section{SVD-Based Universal Spatial Domain Image Steganalysis (2010)}


International Journal of Computer Science, Engineering and Information Technology (IJCSEIT), Vol.2, No.3, June 2012

Gul, G.; Kurugollu[68] proposed steganalyzer models linear dependencies of image rows/columns in local neighborhoods using singular value decomposition transform and employs content independency provided by a Wiener filtering process.

A Hidden Information Blind Detection Method Based on Rough Set Theory (2010)

Wenqiong Yu; Zhuo Li; Lingdi Ping [69] proposed firstly, an improved general steganalysis system framework with practical method and steps; second, the Algorithm based on the rough set theory reduces feature dimension, computational complexity of classification, and eliminates the relevance among statistical features; third, the realization procedure is offered in this algorithm; the SVM classifier is employed to test the spread spectrums steganalysis Cox and Piva.

\section{SURF: Steganalysis Using Random Forests (2010)}

Veena, H.B.; Krishna, S.; Shenoy[70] proposed steganalysis using random forests (SURF) employing HCS (Huffman Code Statistics) features and FR Index (ratio of File size to Resolution). The SURF algorithm proves random forest to be an efficient classifier for steganalysis.

Research on Simulated Annealing Clustering Algorithm in the Steganalysis of Image Based on the One-Class Support Vector Machine(2010)

Peng Luo; Yang Su[71] implemented One-Class Support Vector Machine(OC-SVM) with Simulated Annealing clustering algorithm for steganalysis. The performance of sample clustering is concerned in the OC-SVM with multi-sphere. In previous work, the K-means is mainly used to create such multi-sphere by clustering. But the traditional K-means depends on initial clustering centers and ends local minimum value. So, to solve the problem caused by K-means, the Simulated Annealing is employed into the proposed scheme, which can create more reasonable multi-sphere by finding global optimum solutions in the clustering process.

\section{Towards Genetic Feature Selection in Image Steganalysis (2010)}

Ramezani, M.; Ghaemmaghami, S.[72] compared Fisher linear discriminant, Gaussian naive Bayes, multilayer perceptron, and $\mathrm{k}$ nearest neighbor for steganalysis of suspicious images. The method exploits statistics of the histogram, wavelet statistics, amplitudes of local extrema from the ID and 2D adjacency histograms, center of mass of the histogram characteristic function and co-occurrence matrices for feature extraction process. In order to reduce the proposed features dimension and select the best subset, genetic algorithm is used and the results are compared through principle component analysis and linear discriminant analysis.

\section{Feature Based Steganalysis Using Wavelet Decomposition and Magnitude Statistics (2010)}

T., Gireesh Kumar; R., Jithin; Shankar, Deepa D.[73] compared the efficiency of two embedding algorithms using the image features that are consistent over a wide range of cover images, but are distributed by the presence of embedded data. Image features were extracted after wavelet decomposition of the given image. These features were then given to a SVM classifier to identify the stego content.

\section{Comparison Between Neural Network Steganalysis and Linear Classification Method Stegdetect (2010)}

Holoska, J.; Oplatkova, Z.; Zelinka, I.; Senkerik, R.; Fac.[74] compared universal neural network classification and Stegdetect - a linear classification tool. The results show that neural networks were better than the linear classification tool. 
Blind Image Steganalysis via Joint Co-occurrence Matrix and Statistical Moments of Contourlet Transform (2010)

Sheikhan, M.; Moin, M.S.; Pezhmanpour, M. [75] extracted the features from Contourlet coefficients and co occurrence metrics of subband images. To reduce the number of features, Analysis of Variance (ANOVA) method is used and the selected features are fed to nonlinear Support Vector Machine (SVM) for classification.

\section{Steganalysis Scheme Using the Difference Image of Calibrated Sub-sampling (2010)}

Jeong-Chun Joo; Tae-Woo Oh; Jung-Ho Choi; Heung-Kyu Lee [76] proposed a steganalysis scheme using the difference histogram and image calibration. The message embedding makes the correlation with the neighboring pixels weakens and the random changes of the pixel values generate the block effects among the pixels. The proposed method crops a suspicious image by 1 pixel in a row or column direction and compares the difference histograms. Two distance measures (Manhattan and Euclidean distance) are employed to evaluate the gap between the histograms and then a support vector machine is used as classifier.

\section{Bhattacharyya Distance for Blind Image Steganalysis (2010)}

$\mathrm{Ke} \mathrm{Ke}$; Tao Zhao; Ou Li [77] explores Bhattacharyya Distance principle and introduces it to recognize stego algorithms in use. First of all, we select the most important features by the means of applying Bhattacharyya distance. Then, BP neural network is used to classify cover and stego images.

\section{Universal Steganographic Detection Algorithm in JPEG Image Using the Data-Dependent Kernel (2010)}

Chen Qunjie; Zhong Shangping [78] proposed a steganographic detection method for JPEG image that base on the data-dependent concept, first obtain the initial classifier by SVM training, then the kernel function is modified with conformal transformation by using the information of Support Vectors, retrain with the new kernel to enlarge the spacing around classification boundary, iterate until getting the best result.

\section{HUBFIRE-A Multiclass SVM Based JPEG Steganalysis Using HBCL Statistics and Fr Index (2010)}

Bhat, V.H.; Krishna, S.; Shenoy, P.D.; Venugopal, K.R.; Patnaik, L.M.;[79] proposed new features for JPEG blind steganalysis using a combination of Huffman Bit Code Length (HBCL) Statistics and File size to Resolution ratio (FR Index); the Huffman Bit File Index Resolution (HUBFIRE) algorithm proposed uses these functionals to build the classifier using a multi-class Support Vector Machine (SVM).

\section{An Image Steganalysis Method Based on Characteristic Function Moments and PCA (2011)}

Li Hui; Sun Ziwen; Zhou Zhiping[80] proposed the scheme based on the characteristic function (CF) moments of three-level wavelet subbands including the further decomposition coefficients of the first scale diagonal subband. The first three statistical moments of each wavelet band of test image and prediction-error image are selected to form 102 dimensional features for steganalysis. Principal Components Analysis (PCA) is utilized to reduce the features and the support vector machine (SVM) is adopted as the classifier. 


\section{Blind Steganalysis with High Generalization Capability for Different Image Databases Using L-GEM (2011)}

Ng, Wing W. Y.; He, Zhi-Min; Chan, Patrick P. K.; Yeung, Daniel S.[81] expanded the Markov features and apply L-GEM based neural network in our method to enhance the generalization capability of steganalysis.

\section{A Universal Steganalysis to Steganographic Images on Frequency Domain (2011)}

Ping, Qian; Li-ya, Chen; Meng [82] the detection is achieved based on the spectrum analysis of difference histogram of frequency coefficients according to evident spectrum difference between non-steg-images and steg-images. The physical frame of the algorithm is easy and the detection method can be put into practice. Experimental results from detecting steganographic images of DCT domain and DWT domain show that the detection performance is satisfying.

\section{SUMMARY}

In this paper, many of the important blind Steganalysis techniques have been presented and analyzed. The two steps are done in every paper i.e. features extraction and pattern classification.

\section{Features Extraction Techniques and Pattern Classification Techniques are based on:}

(1) 2-D Markov chain of threshold prediction-error image, learning vector quantization (LVQ), statistical analysis of empirical matrix (EM), data fusion technique, high-order wavelet decomposition to capture statistical difference.

(2) Multidirectional Markov models of DCT coefficients, statistical moments of histogram of multi-level wavelet subbands in frequency domain, sub-band coefficients mean squared error, Robert gradient energy in pixel domain, variance of Laplacian parameter in DCT(discrete cosine transform) domain and higher-order statistics extracted from wavelet coefficients.

(3) Intra- and inter- color correlations of wavelet coefficients, moments in frequency domain of wavelet sub-band's histogram, forward difference in three directions, horizontal, vertical and diagonal, co-occurrence matrix.

(4) Wavelet Packet Transform(WPT), image run-length histograms, statistical moments of wavelet characteristic function (SMWCF), 1D statistical grayscale histogram, Bhattacharyya distance, intra-block and inter-block quantized DCT (discrete cosine transform) coefficients by the Markov empirical transition matrices.

(5) Generalized Gaussian Distribution (GGD), difference of the expanded Markov features, polynomial fitting features on the histogram of the DCT coefficients, features from the luminance and chrominance components of the images, the characteristic function and the center of mass (COM), two-level Haar WPD, PDF, CF and Absolute moment calculated from wavelet multi-resolution.

(6) Characteristic function moments of three-level wavelet subbands, subset of candidate transformations, nonlinear dimensionality reduction method (ISOMAP), forward selection algorithm with F-score, MWCF and F-score method called as FS-MWCF, alpha-trimmed mean, fractional Fourier transform (FRFT), transition probability matrix, LogSv, datamining tool.

(7) SADRID-I, Radial Basis Function Neural Network (RBFNN), HCS (Huffman Code Statistics), FR Index (ratio of File size to Resolution), Gaussian naive Bayes, Analysis of Variance (ANOVA), Manhattan and Euclidean distance, Huffman Bit Code Length (HBCL), Huffman Bit File Index Resolution (HUBFIRE), spectrum analysis of difference histogram of frequency coefficients. 
(8) Support Vector Machine (SVM), class-wise non-principal components analysis (CNPCA), BP(back propagation) neural network, Support vector machines (SVM) with RBF kernel, back-propagation (BP) neural network, artificial neural network (ANN), neuro-fuzzy inference system, genetic algorithm, Fisher linear discriminator (FLD), NaiA $\hat{A}_{i}$ ve Bayes classifier, Decision Tree Classifier and K-nearest neighbor, logistic regression model, Bayesian network model, One-Class Support Vector Machine(OC-SVM), L-GEM based neural network.

\section{REFERENCE}

[1] Kelly, J., “Terror Groups Hide Behind Web Encryption”, USA Today, 2 May 2001, http:// www. usatoday.com /life/cyber/tech/2001-02-05-binladen.htm.

[2] Anonymus "What is Steganography?" www.tech-faq.com/steganography.html.

[3] Apostol Maile,Lasun Zhanna,Sardinas Ana,Yigit Yasemin ," Image Encryption Using LSB/MSB “, Term Project, CpE-462, 04/30/02,April 2002.

[4] Anonymus "Miscellaneous Steganographic" ,scien.stanford.edu/class/ psyh221/project /05/vvikram/stegomisc.htm.

[5] Jahne,Bernd,Digital Image Processing, "Concepts,Algorithms ,an Scientific Applications" II nd ed.,Springer-Verlag, 1993.

[6] R. B. Wolfgang, E. J. Delp ,"A watermark for digital Images", in International Conference on Images Processing,Lausanne,Switzerland, , IEEE, pp. 219-222, 16-19 Sept. 1996.

[7] Mehdi Kharrazi1, Husrev T. Sencar, and Nasir Memon,'Image Steganography:Concepts and Practices" Department of Electrical and Computer Engineering,Department of Computer and Information Science Polytechnic University, Brooklyn, NY 11201,USA fmehdi taha, memong@isis.poly.edu,www.ims.nus.edu.sg/preprints/2004-5.pdf.

[8] Swagota Bera and Monisha Sharma,"Survey on Steganographic Techniques \&Steganalysis", National Conference in Advances in electronics \& Telecommunication Technologia,vision2020,IIM,28-29, Pune, India, Oct 2007.

[9] Monisha Sharma, Swagota Bera , "Steganalysis of Real Time Image by Statistical Attacks", International Journal of Engineering Science and Technology,Vol. 2(9), 4397- 4406, 2010.

[10] Monisha Sharma, Swagota Bera , "Steganalysis of the Image by Visual and Statistical attack", in iMa nager's Journal of Electronics Engineering,ISSN-2229-7286,Vol.1,No.2,Dec -2010,Feb-2011,P49-55.

[11] Steganalysis based on Markov Model of Thresholded Prediction-Error Image Zou, D.; Shi, Y.Q.; Wei Su; Guorong Xuan; Multimedia and Expo, 2006 IEEE International Conference on 9-12 July 2006 Page(s): 1365 - 1368.

[12] Steganalysis Embedding Percentage Determination with Learning Vector Quantization Rodriguez, B.M.;Peterson, G.L.;Bauer,K.W.;Agaian,S.S.;Systems, Man and Cybernetics, 2006. SMC '06. IEEE International Conference on Volume 3, 8-11 Oct. 2006 page(s): 1861 - 1865.

[13] Blind Image Steganalysis Based on Statistical Analysis of Empirical Matrix Xiaochuan Chen; Yunhong Wang; Tieniu Tan; Lei Guo;Pattern Recognition, 2006. ICPR 2006,18th International Conference on Volume 3, 0-0 0 Page(s):1107 - 1110.

[14] Spatial-frequency Feature Vector Fusion Based Steganalysis Hong Cai; Agaian, S.S.;Systems, Man and Cybernetics, 2006. SMC '06. IEEE International Conference on Volume 3, 8-11 Oct. 2006 Page(s): 1866 - 1870 .

[15] Blind Steganalysis using Wavelet Statistics and ANOVA Shuang-Huan Zhan; Hong-Bin Zhang; Machine Learning and Cybernetics, 2007 International Conference on Volume 5,19-22 Aug. 2007 Page(s):2515 - 2519 .

[16] Passive Steganalysis Using Image Quality Metrics and Multi-class Support Vector Machine Bo Xu; Jiazhen Wang; Xiaqin Liu; Zhe Zhang;Natural Computation, 2007, ICNC 2007. Third International Conference on Volume 3, 24-27 Aug. 2007 Page(s): 215 - 220 .

[17] JPEG Steganalysis Based on Classwise Non-Principal Components Analysis and Multi-Directional Markov Model Guorong Xuan; Xia Cui; Shi, Y.Q.; Wen Chen; Xuefeng Tong;Cong Huang; Multimedia and Expo, 2007 IEEE International Conference on 2-5 July 2007 Page(s):903 - 906.

[18] A JPEG Steganalysis Algorithm with Embedded Rate Estimation Based on Multi-class SVM Pan, Xiaosheng; Image and Signal Processing, 2008. CISP '08. Congress on Volume 5, 27-30 May 2008 Page(s):613 - 615 . 
[19] Image Steganalysis Based on Statistical Moments of Wavelet Subband Histograms in Different Frequencies and Support Vector Machine Mehrabi, M.A.; Faez, K.; Bayesteh, A.R.;Natural Computation, 2007. ICNC 2007. Third International Conference on Volume 1, 24-27 Aug. 2007 Page(s):587 - 590.

[20] A Steganalysis Method Based On Quantization Attack Huang, Jifeng; Jiao, Qingju;Image and Signal Processing, 2008. CISP '08. Congress on Volume 5, 27-30 May 2008 Page(s):40 - 644.

[21] A blind image steganalysis based on features from three domains Yuan Liu; Li Huang;Ping Wang; Guodong Wang;Control and Decision Conference, 2008. CCDC 2008, Chinese ,2-4 July 2008 Page(s):2933 - 2936.

[22] JPEG steganalysis using color correlation and training on clean images only Hong Cai;Agaian,S.S.;Machine Learning and Cybernetics, 2008 International Conference on Volume 7, 12 -15 July 2008 Page(s):3710 - 3713.

[23] A New Steganalysis Method Using High-pass Filter for JPEG Image Han-ling Zhang;Shu-yi Wang; Electronic Commerce and Security,2008 International Symposium on 3-5 Aug. 2008 Page(s):165 168.

[24] Steganalysis Based on Co-occurrence Matrix of Differential Image Ziwen Sun; Maomao Hui; Chao Guan;Intelligent Information Hiding and Multimedia Signal Processing, 2008,IIHMSP '08 International Conference on 15-17 Aug. 2008 Page(s):1097 - 1100.

[25] Steganalysis of LSB Matching Based on Co-occurrence Matrix and Removing Most Significant Bit Planes Abolghasemi, M.;Aghainia,H.; Faez, K.; Mehrabi, M.A.;Intelligent Information Hiding and Multimedia Signal Processing, 2008. IIHMSP '08 International Conference on 15-17 Aug. 2008 Page(s): 1527 - 1530 .

[26] Image universal steganalysis based on wavelet packet transform Xiangyang Luo; Fenlin Liu; Jianming Chen; Yining Zhang; Multimedia Signal Processing, 2008 IEEE 10th Workshop on Digital 2008 , Page(s): 780 - 784 .

[27] Features-Pooling Blind JPEG Image SteganalysisChiew Kang Leng; Pieprzyk, J.;Computing: Techniques and Applications, 2008. DICTA '08.Digital Image1-3 Dec. 2008 Page(s):96-103.

[28] Blind image steganalysis based on run-length histogram analysis Jing Dong; Tieniu Tan;Image Processing, 2008. ICIP 2008. 15th IEEE International Conference on Digital Object Identifier: 10.1109/ICIP.2008.4712192 Publication Year: 2008 , Page(s): $2064-2067$.

[29] Detection of BPCS-steganography using SMWCF steganalysis and SVM Lopez- Hernandez, Julio; Martinez-Noriega, Raul; Nakano-Miyatake, Mariko; Yamaguchi, Kazuhiko;Information Theory and Its Applications, 2008. ISITA 2008. International Symposium on 7-10 Dec. 2008 Page(s):1 - 5 .

[30] Steganalysis of LSB matching based on histogram features in grayscale image Xu Mankun; Li Tianyun; Ping Xijian;Communication Technology, 2008. ICCT 2008. 11th IEEE International Conference on 10- 12 Nov. 2008 Page(s):669 - 672.

[31] A New Blind Steganalysis Method for JPEG Images Xiang Li; Jianhua Li;Computer Science and Software Engineering, 2008 International Conference on Volume 3,12-14 Dec. 2008 Page(s):939942 .

[32] Multi-class steganalysis for Jpeg stego algorithms Ping Wang; Fenlin Liu; Guodong Wang; Yifeng Sun; Daofu Gong;Image Processing, 2008. ICIP 2008. 15th IEEE International Conference on 1215 Oct. 2008 Page(s):2076 - 2079 .

[33] Universal JPEG steganalysis based on microscopic and macroscopic calibration Fangjun Huang; Jiwu Huang; Bin Li;Image Processing, 2008. ICIP 2008. 15th IEEE International Conference on 1215 Oct. 2008 Page(s):2068 - 2071 .

[34] Detect Information-Hiding Type and Length in JPEG Images by Using Neuro-fuzzy Inference Systems Liu, Qingzhong; Sung, Andrew H.; Image and Signal Processing,2008. CISP '08. Congress on Volume: 5 ,2008 , Page(s): 692 - 696 .

[35] Steganalysis of multi-class JPEG images based on expanded Markov features and polynomial fitting Qingzhong Liu; Sung, A.H.; Ribeiro, B.M.; Ferreira, R.; Neural Networks, 2008. IJCNN 2008. (IEEE World Congress on Computational Intelligence).IEEE International Joint Conference ,2008 , page(s): 3352 - 3357 .

[36] Universal Steganalysis Using Color Correlation and Feature Fusion Yuan-lu Tu; Sheng- rong Gong; Information Science and Engineering, 2008. ISISE '08. International Symposium on Volume: 1, Publication Year: 2008 , Page(s): 107 - 111.

[37] An Investigation of Genetic Algorithm on Steganalysis Techniques Xiao Yi Yu; Aiming Wang; Intelligent Information Hiding and Multimedia Signal Processing, 2009. IIH-MSP '09. Fifth International Conference ,2009, Page(s): 1118 - 1121 . 
[38] Feature-Based Steganalysis for JPEG Images Zhuo Li; Kuijun Lu; Xianting Zeng; Xuezeng Pan; 2009, Page(s): 76 - 80 .

[39] Universal Image Steganalysis Based on Wavelet Packet Decomposition and Empirical Transition Matrix in Wavelet Domain Xiaoyuan Yang; Yu Lei; Xiaozhong Pan; Jia Liu; Computer ScienceTechnology and Applications, 2009. IFCSTA '09. International Forum on Volume: 2 ,2009 , Page(s): $179-182$.

[40] Intelligent detection of LSB stego anomalies in images using soft computing paradigms Geetha, S.; Sindhu, S.S.; Ishwarya, N.; Mohan, A.; Amuthayazhini, P.; Kamaraj, N.; Methods and Models in Computer Science, 2009. ICM2CS 2009. Proceeding of International Conference on Publication Year: 2009 , Page(s): $1-5$.

[41] An Image Steganalysis Method Based on Characteristic Function Moments of Wavelet Subbands Ziwen Sun; Hui Li; Zhijian Wu; Zhiping Zhou; Artificial Intelligence and Computational Intelligence, 2009. AICI '09. International Conference on Volume: 1, 2009, Page(s): 291 - 295.

[42] JPEG image steganalysis method based on binary similarity measures Jing-Qu Lin; Shang-Ping Zhong; Machine Learning and Cybernetics, 2009 International Conference on Volume: 4 ,2009, Page(s): 2238 - 2243.

[43] Steganalysis Based on Regression Model and Bayesion Network Xiao Yi Yu; Aiming Wang; Multimedia Information Networking and Security, 2009. MINES '09. international Conference on Volume: 1 ,2009, Page(s): 41 - 44 .

[44] Steganalysis for JPEG images based on manifold learningXiaomei Quan; Hongbin Zhang; Applications of Digital Information and Web Technologies, 2009. ICADIWT '09. Second International Conference, 2009 , Page(s): 707 - 712 .

[45] Using Higher Order DCT Difference to Effective Improve Markov Process Based JPEG Steganalysis Detection Rate Guibin Yang; Hongbin Zhang; Information Processing,2009. APCIP 2009. AsiaPacific Conference on Volume: 2,2009 , Page(s): 47 - 50 .

[46] Reduction of Markov Extended Features in JPEG Image Steganalysis Jing-qu Lin; Xiao-dong Wang; Shang-ping Zhong; Image and Signal Processing, 2009. CISP '09. 2nd International Congress on , 2009 , Page(s): $1-5$.

[47] Steganalysis on Character Substitution Using Support Vector Machine Xinxin Zhao; Liusheng Huang; Lingjun Li; Wei Yang; Zhili Chen; Zhenshan Yu; Knowledge Discovery and Data Mining, 2009. WKDD 2009. Second International Workshop on 2009, Page(s): $84-88$.

[48] Universal Steganalysis Based on Statistical Models Using Reorganization of Block- based DCT Coefficients Shaohui Liu; Lin Ma; Hongxun Yao; Debin Zhao; Information Assurance and Security, 2009. IAS '09. Fifth International Conference on Volume: 1,2009 , Page(s): 778 - 781 .

[49] Steganalysis Based on Bayesion Network and Genetic Algorithm Xiao Yi Yu; Aiming Wang; Image and Signal Processing, 2009. CISP '09. 2nd International Congress on 2009, Page(s): 1 - 4 .

[50] Blind steganalysis method for BMP images based on statistical MWCF and F-score method Xue Zhang; Shang-Ping Zhong; Wavelet Analysis and Pattern Recognition, 2009. ICWAPR 2009. International Conference on ,2009, Page(s): 442 - 447.

[51] A Methodology of Steganalysis for Images Hernandez-Chamorro, A.; Espejel-Trujillo, A.; LopezHernandez, J.; Nakano-Miyatake, M.; Perez-Meana, H.; Electrical, Communications, and Computers, 2009. CONIELECOMP 2009. International Conference on 2009, Page(s):102 - 106.

[52] Alpha-trimmed image estimation for JPEG steganography detection Mei-Ching Chen; Agaian, S.S.; Chen, C.L.P.; Rodriguez, B.M.; Systems, Man and Cybernetics, 2009. SMC 2009. IEEE International Conference on 2009, Page(s): 4581 - 4585.

[53] Blind steganalysis based on features in fractional Fourier transform domain Chao-en Zhou; Jiu-chao Feng; Yi-xian Yang; Communications, Circuits and Systems, 2009. ICCCAS 2009. International Conference on 2009, Page(s): $301-303$.

[54] Blind JPEG Steganalysis Based on Correlations of DCT Cofficients in Multi-directions and Calibrations Wang Yong; Liu JiuFen; Zhang WeiMing; Multimedia Information Networking and Security, 2009. MINES '09. International Conference on Volume: 1,2009 , Page(s): 495 - 499.

[55] A novel universal steganalyser design: "LogSv" Gul, G.; Kurugollu, F.; Image Processing (ICIP), 2009 16th IEEE International Conference on 2009 , Page(s): 4249 - 4252.

[56] Steganalysis for Markov Feature of Difference Array in DCT Domain Zhiping Zhou; Maomao Hui; Fuzzy Systems and Knowledge Discovery, 2009. FSKD '09. Sixth International Conference on Volume: 7,2009 , Page(s): 581 - 584 .

[57] A Universal Steganalysis Using Features Derived from the Differential Image Histogram in Frequency Domain Deng Qian-lan; Lin Jia-jun; Image and Signal Processing, 2009. CISP '09. 2nd International Congress on 2009, Page(s): 1 - 4 . 
[58] Universal Steganalysis to Images with WBMC Model Xiaoyuan Yang; Shifeng Wang; Jia Liu; Information Assurance and Security, 2009. IAS '09. Fifth International Conference on Volume: 2, 2009, Page(s): 627 - 630 .

[59] Blind detection for JPEG steganography Wenqiong Yu; Zhuo Li; Lingdi Ping; Networking and Information Technology (ICNIT), 2010 International Conference on 2010, Page(s): 128 - 132 .

[60] Statistical steganalyis of images using open source software Kaipa, B.; Robila, S.A.; Applications and Technology Conference (LISAT), 2010 Long Island Systems, 2010 , Page(s): 1 - 5 .

[61] Block-based image steganalysis: Algorithm and performance evaluation Seongho Cho; Byung-Ho Cha; Jingwei Wang; Kuo, C.-C.J.; Circuits and Systems (ISCAS), Proceedings of 2010 IEEE International Symposium 2010, Page(s): 1679 - 1682 .

[62] Block-based image steganalysis for a multi-classifier Seongho Cho; Jingwei Wang; Kuo, C.-C.J.; Byung-Ho Cha; Multimedia and Expo (ICME), 2010 IEEE International Conference on 2010, Page(s): $1457-1462$.

[63] An Ensemble of Classifiers Approach to Steganalysis Bayram, S.; Dirik, A.E.; Sencar, H.T.; Memon, N.;Pattern Recognition (ICPR), 2010 20th International Conference on 2010, Page(s): 4376 - 4379.

[64] Steganalysis Algorithm Based on the D Reduction of Improved Differential Matrix in Images Wenqiong Yu; Zhuo Li; Lingdi Ping; Pattern Recognition (CCPR), 2010 Chinese Conference on 2010, Page(s): 1 - 7 .

[65] The blind detection of information hiding in color image Deng Qian-lan; Computer Engineering and Technology (ICCET), 2010 2nd International Conference on Volume: 7,2010,Page(s):V7-346-V7348.

[66] Steganalytic attack for an adaptive steganography using support vector machine Yamini, B.; Sabitha, R.;Emerging Trends in Robotics and Communication Technologies (INTERACT), 2010 International Conference on 2010 , Page(s): 56 - 58 .

[67] Steganography detection using localized generalization error model Zhi-Min He; Ng, W.W.Y.; Chan, P.P.K.; Yeung, D.S.; Systems Man and Cybernetics (SMC), 2010 IEEE International Conference on 2010, Page(s): 1544 - 1549.

[68] SVD-Based Universal Spatial Domain Image Steganalysis Gul, G.; Kurugollu, F.; Information Forensics and Security, IEEE Transactions on Volume: 5 , Issue: 22010 , Page(s): 349 - 353.

[69] A Hidden Information Blind Detection Method Based on Rough Set Theory Wenqiong Yu; Zhuo Li; Lingdi Ping; Pattern Recognition (CCPR), 2010 Chinese Conference on 2010 , Page(s): 1 - 5.

[70] SURF: Steganalysis using random forests Veena, H.B.; Krishna, S.; Shenoy, P.D.; Intelligent Systems Design and Applications (ISDA), 2010 10th International Conference on 2010, Page(s): 373-378.

[71] Research on Simulated Annealing clustering algorithm in the steganalysis of image based on the One-Class Support Vector Machine Peng Luo; Yang Su; Computer Application and System Modeling (ICCASM), 2010 International Conference on Volume: 142010 , Page(s): V14-446 - V14450 .

[72] Towards Genetic Feature Selection in Image Steganalysis Ramezani, M.; Ghaemmaghami, S.; Consumer Communications and Networking Conference (CCNC), 2010 7th IEEE 2010 ,Page(s): 14.

[73] Feature Based Steganalysis Using Wavelet Decomposition and Magnitude Statistics T., Gireesh Kumar; R., Jithin; Shankar, Deepa D.; Advances in Computer Engineering (ACE), 2010 International Conference on 2010, Page(s): 298 - 300 .

[74] Comparison between Neural Network Steganalysis and Linear Classification Method Stegdetect Holoska, J.; Oplatkova, Z.; Zelinka, I.; Senkerik, R.; Computational Intelligence, Modelling and Simulation (CIMSiM), 2010 Second International Conference on 2010 , Page(s): 15 - 20 .

[75] Blind image steganalysis via joint co-occurrence matrix and statistical moments of contourlet transform Sheikhan, M.; Moin, M.S.; Pezhmanpour, M.; Intelligent Systems Design and Applications (ISDA), 2010 10th International Conference on 2010, Page(s): 368 - 372 .

[76] Steganalysis Scheme Using the Difference Image of Calibrated Sub-sampling Jeong-Chun Joo; TaeWoo Oh; Jung-Ho Choi; Heung-Kyu Lee;Intelligent Information Hiding and Multimedia Signal Processing (IIH-MSP), 2010 Sixth International Conference on 2010, Page(s): 51 - 54 .

[77] Bhattacharyya Distance for Blind Image Steganalysis Ke Ke; Tao Zhao; Ou Li; Multimedia Information Networking and Security (MINES), 2010 International Conference on 2010, Page(s): 658-661 .

[78] Universal Steganographic Detection Algorithmin in JPEG Image Using the Data-Dependent Kernel Chen Qunjie; Zhong Shangping; Electronic Commerce and Security (ISECS), 2010 Third International Symposium on 2010, Page(s): 232 - 236. 
International Journal of Computer Science, Engineering and Information Technology (IJCSEIT), Vol.2, No.3, June 2012

[79] HUBFIRE - A multi-class SVM based JPEG steganalysis using HBCL statistics and Fr Index Bhat, V.H.; Krishna, S.; Shenoy, P.D.; Venugopal, K.R.; Patnaik, L.M.; Security and Cryptography (SECRYPT), Proceedings of the 2010 International Conference on Publication Year: 2010, Page(s):16.

[80] An image steganalysis method based on characteristic function moments and PCA Li Hui; Sun Ziwen; Zhou Zhiping; Control Conference (CCC), 2011 30th Chinese Publication Year: 2011 , Page(s): 3005 - 3008 .

[81] Blind steganalysis with high generalization capability for different image databases using L-GEM Ng,Wing W. Y.; He, Zhi-Min; Chan, Patrick P. K.; Yeung, Daniel S.; Machine Learning and Cybernetics (ICMLC), 2011 International Conference on Volume: 4 ,2011, Page(s): 1690 - 1695.

[82] A universal steganalysis to steganographic images on frequency domain Ping, Qian; Li-ya,Chen; Meng,Wu;E-Business and E -Government (ICEE), 2011 International Conference on,2011, Page(s):1-5. 